\title{
One-year follow-up in patients with brainstem infarction due to large-artery atherothrombosis
}

This article was published in the following Dove Press journal:

Neuropsychiatric Disease and Treatment

18 February 2015

Number of times this article has been viewed

\author{
Mehmet Kolukısa' \\ Tugce Ozdemir Gültekin' \\ Gozde Eryigit Baran' \\ Ayse Aralasmak ${ }^{2}$ \\ Gülsen Kocaman' \\ Azize Esra Gürsoy' \\ Talip Asil' \\ 'Department of Neurology, \\ ${ }^{2}$ Department of Radiology, Faculty \\ of Medicine, Bezmialem Vakıf \\ University, Istanbul, Turkey
}

\begin{abstract}
Background: Posterior circulation infarction accounts for approximately $25 \%$ of the ischemic strokes. A number of different conditions may be associated with the development of brainstem infarction. Prognosis and recurrence rate of brainstem infarction due to large-artery atherothrombosis is still controversial.
\end{abstract}

Methods: A total of 826 patients with ischemic stroke were admitted to our clinic during a 15 -month period. Patients with clinical and radiological evidence of brainstem infarction were comprehensively assessed with appropriate vascular imaging modalities and for cardiological causes. Subjects with an established diagnosis of large-artery atherothrombosis were followed up for 1 year in terms of prognosis and recurrence rates.

Results: Of the 101 patients with an established brainstem infarction, the diagnostic work-up indicated the presence of large-artery atherothrombosis as the causative factor in 53 . A recurrent stroke was detected in the posterior circulation within the first 3 months in $5.8 \%$ and within 1 year in $9.8 \%$ of these individuals. The 1 -year mortality rate was $11.7 \%$. All patients with recurrent stroke had intracranial vascular narrowing at baseline.

Conclusion: Our findings demonstrate a particularly high rate of recurrence and mortality at 1 year among patients who have a brainstem infarction due to intracranial arterial stenosis.

Keywords: posterior circulation, intracranial arteries, extracranial arteries, stroke, vertebral artery, basilar artery

\section{Background}

Posterior circulation infarction (POCI) syndrome is the involvement of brainstem, cerebellum, and occipital lobes supplied by the vertebrobasilar arterial system. POCI is recognized by the presence of visual field defects, brainstem findings, or cerebellar signs or by any combination of these manifestations as a result of the proximal or distal occlusion of the arteries forming the vertebrobasilar system. ${ }^{1} \mathrm{POCI}$ is responsible for approximately $25 \%$ of the ischemic strokes. ${ }^{2}$ Brainstem strokes result from the involvement of the basilar artery or its branches due to embolism from artery to artery or cardioembolism or hemodynamic factors or may present as lacunar syndromes. ${ }^{3}$ It may be challenging to establish a link between the localization of the infarction and etiology, particularly in brainstem involvement, due to the fact that mesencephalon, pons, and bulbus are supplied by different arteries or due to frequent occurrence of normal variations. Conversely, brain areas affected by embolic or hemodynamic infarctions of the anterior circulation are quite well established.

Treatment strategies are well defined in patients with anterior circulation involvement due to large-artery atherothrombosis, particularly in the presence of extracranial arterial stenosis. On the other hand, in patients with brainstem infarction who have large-arterial atherothrombosis, there is a relative lack of information on therapeutic options regardless of the presence of extracranial or intracranial arterial stenosis. ${ }^{4}$
Department of Neurology, Faculty of Medicine, Bezmialem Vakıf University, Vatan Cad 34093, Istanbul, Turkey Tel $+902 I 24531700$

Email talip_asil@yahoo.com 
This study was undertaken to assess the clinical signs and prognosis in patients who presented with brainstem infarction resulting from large-artery atherothrombosis.

\section{Patients and methods}

This study was carried out at the Department of Neurology, Bezmialem Vakıf University, between January 2012 and April 2013 involving 101 patients with brainstem infarction among a total of 826 patients admitted with a diagnosis of ischemic stroke during the same period. Only those with large-artery atherothrombosis were included. The diagnosis, large-artery atherothrombosis, was based on the detection of $\geq 50 \%$ stenosis in intracranial or extracranial vertebral arteries or basilar artery with an association between clinical signs and localization of the stenosis. ${ }^{5}$ Other etiological factors such as dissection or vascular anomalies were excluded. Local ethics committee approval was obtained and patient data were retrospectively retrieved from the Hospital Database of the Medical Faculty of Bezmialem Vakıf University.

Inclusion criteria were as follows: age $>18$ years, presence of brainstem infarction, detection of large-artery atherothrombosis as the etiological explanation for the infarction, having a diffusion magnetic resonance imaging (MRI) within 7 days of the incidence, presence of an imaging study for the vascular tree with magnetic resonance angiography (MRA) or computed tomography angiogram (CTA), and an examination carried out with transesophageal echocardiogram and Holter monitorization for patients who required electrocardiography (ECG) and transthoracic echocardiography for a possible diagnosis of cardioembolism.

Patients with other etiological factors such as bleeding or tumors detected by computed tomography (CT) or MRI, anterior circulation infarcts, (stenotic patients with arterial dissection) posterior infarct without the involvement of the brainstem (eg, those with pure cerebellar or thalamic infarcts), absence of adequate imaging studies, and etiological factors other than large-arterial disease such as small-vessels disease or cardioembolic stroke, were excluded. However, patients with infarcts at different locations with simultaneous involvement of the brainstem were included. Patients who underwent thrombolytic treatment were excluded.

Medical history, neurological examination, routine blood tests, imaging studies (CT+1.5-Tesla MRI), cerebral angiography, and 12-lead ECG results were assessed and recorded in each patient. Patients with metallic valves or prostheses were assessed using vertebral system Doppler examination instead of cerebral MRA.

Age, sex, presence or absence of ischemic stroke, coronary artery disease and/or congestive heart failure, and cardiovascular risk factors were recorded. The latter was defined as follows for study purposes: use of antihypertensive agents, a systolic blood pressure $\geq 140 \mathrm{mmHg}$ or a diastolic blood pressure $\geq 90 \mathrm{mmHg} 2$ weeks before or after the onset of stroke, diabetes status, use of hypoglycemic agents, a casual blood glucose $\geq 200 \mathrm{mg} / \mathrm{dL}$ or a baseline glycosylated hemoglobin $\geq 6.5 \%$, hyperlipidemia, use of antihyperlipidemic agents, or an low density lipoprotein of $\geq 130 \mathrm{mg} / \mathrm{dL}$ at baseline, and use of tobacco.

Diffusion weighted imaging-MRI and cranial CT imaging studies were performed at the emergency room. The localization of the brainstem infarct (bulbus, pons, or mesencephalon) were recorded (Figure 1), as well as other areas involved (eg, cerebellum, occipital lobe, anterior circulation) if present. CTA or MRA was performed in all patients to delineate the anatomy of the vascular tree. Patients with a $\geq 50 \%$ stenosis in vertebral and basilar arteries as detected by CTA/MRA were considered to have large-artery atherothrombosis. For both CTA and MRA, the North American Symptomatic Carotid Endarterectomy Trial (NASCET) criteria was used to assess the severity of the stenosis in extracranial vessels ${ }^{6}$ and the severity of the stenosis in intracranial vessels was estimated using the method described in the Warfarin-Aspirin Symptomatic Intracranial Disease trial. $^{7}$ All disagreements of greater than $10 \%$ were reviewed by a third reader who decided between the two measurements made. The localization of the stenosis and other accompanying stenotic lesions in the anterior circulation were also recorded if present (Figures 2 and 3).

Clinical presentation, including vertigo, ataxia, gaze paresis, and dysarthria, were recorded as well as the National

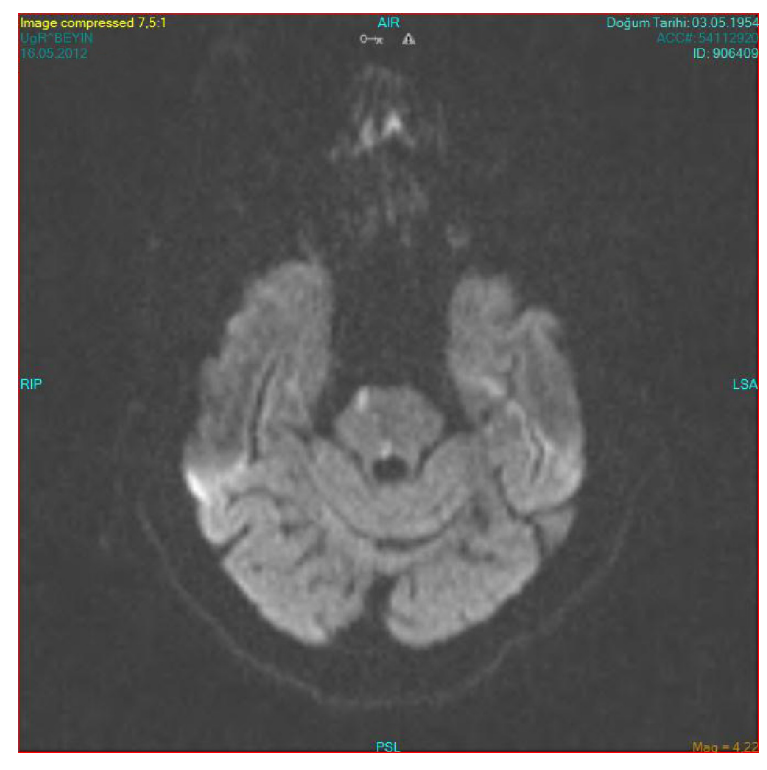

Figure I Multiple pons infarcts in magnetic resonance images on diffusion-weighted images. 


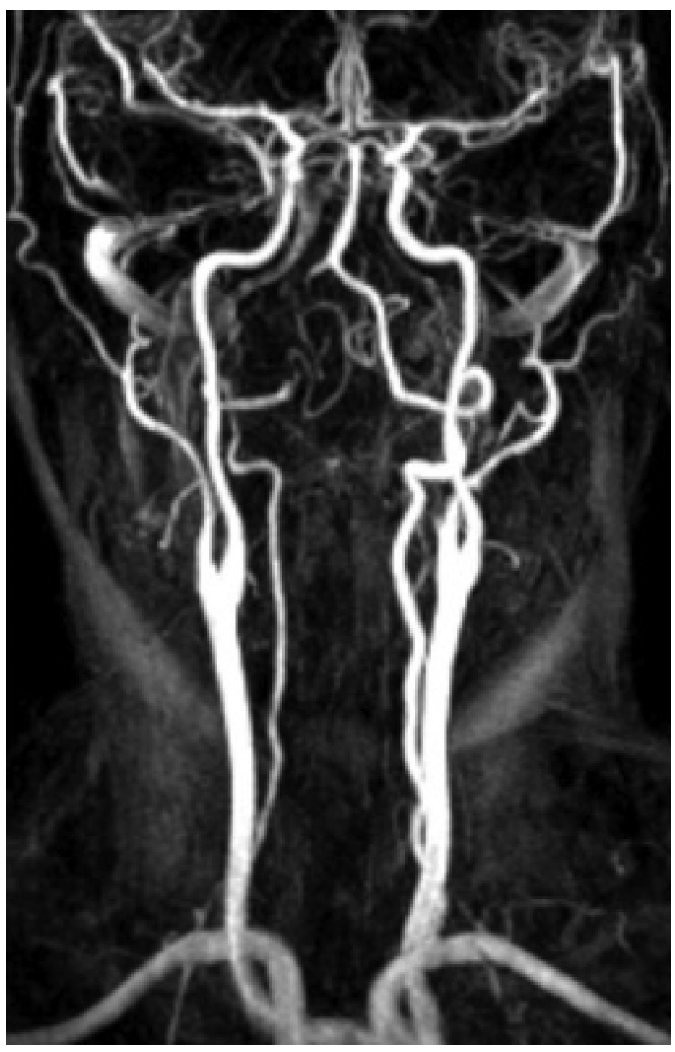

Figure 2 Right V4 stenosis on cervical magnetic resonance angiography.

Institutes of Health (NIH) scores at the time of emergency room visit. Treatments that were being administered at the time of discharge were recorded. Modified Rankin scores at 3 months were calculated either by face-to-face interviews at the outpatient unit or by telephone calls. The time and causes of death were also recorded. Hospital admissions for any

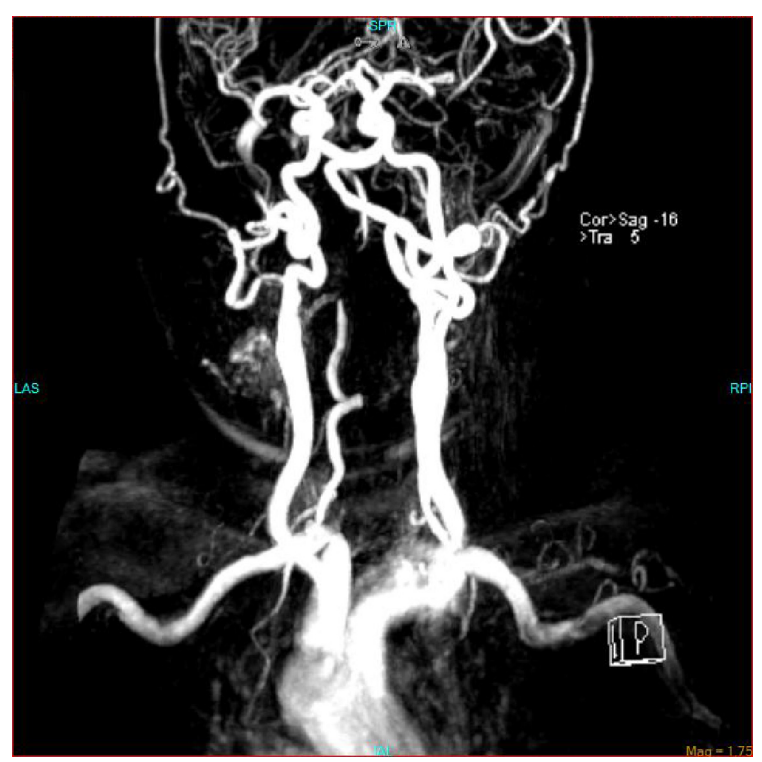

Figure 3 Right V2 stenosis on cervical magnetic resonance angiography. reason and the localization and timing of recurrent strokes occurring during the 1-year follow-up were determined. Kaplan-Meier curve analysis has been used for survival. Fisher's exact test was used to examine the prognostic variables for recurrent strokes and mortality.

\section{Results}

A total of 53 patients with a mean age of $65.3 \pm 1.22$ years who had brainstem infarction with large-vessel atherothrombosis were recruited into the study. Of the study participants, 30 were males. The risk factors in our patient group are shown in Table 1. At the time of initial presentation, 24 patients had ataxia, 20 had dysarthria, four had gaze paresis, and 22 had paresis with an overall mean NIH score of $7.9 \pm 2.8$. Also, two of the 53 subjects had impaired consciousness. Anesthesia was not administered, and no patients underwent endovascular or other type of surgical intervention.

MR diffusion imaging was performed in all patients within the first 7 days of the incidence showing limited diffusion consistent with acute ischemia at pons, mesencephalon, and bulbus in 30, five, and 18 patients, respectively. Furthermore, three patients had thalamic, nine had cerebellar, and three had occipital infarcts additionally.

Forty patients underwent an MRA examination, nine underwent a CTA, and four had both. $\mathrm{A} \geq 50 \%$ stenosis was detected at the extracranial vertebral artery in 13, at intracranial vertebral artery in 31, and at basilar artery in eleven patients. Angiographic examination revealed concurrent stenosis in intracranial vertebral artery and basilar artery in four patients. Two patients had both extracranial and intracranial stenosis.

The average modified Rankin score estimated 7 days after the onset of stroke or on the day of discharge was 3.09 \pm 1.14 . The corresponding modified Rankin scores at 3 months and 1 year after discharge were $1.98 \pm 1.48$ and $1.7 \pm 1.9$, respectively. At the time of discharge, among 53 patients 22 were prescribed a mono-antiplatelet therapy (20 acetylsalicylic acid [ASA], two clopidogrel), and 31 were prescribed a dual-antiplatelet therapy (ASA + clopidogrel). Similarly, of the 53 patients, 33 were on statins at the time of discharge, while 40 were receiving antihypertensive agents.

Table I Demographic factors

\begin{tabular}{ll}
\hline Risk factors & n (\%) \\
\hline Hypertension & $42(79.2)$ \\
Diabetes mellitus & $34(64.1)$ \\
Hyperlipidemia & $33(62.2)$ \\
Cigarette smoking & $17(32.0)$ \\
Coronary artery disease & $12(22.6)$ \\
Previous stroke & $7(13.2)$ \\
\hline
\end{tabular}


A total of 51 patients could be contacted at 1-year follow-up, and recurrent strokes had occurred in the posterior circulation in five cases. The earliest recurrence occurred in two patients at 45 days, while for others recurrence occurred at 3, 8, and 9 months, respectively. In all patients with a recurrence, intracranial arterial narrowing was present at baseline.

Of the 51 patients with a 1-year follow-up six had died, with the earliest death occurring on day 45. Four deaths were recorded after the development of recurrent stroke, while one patient died due to myocardial infarction, and one patient due to undetermined causes. In all the patients who died, the initial arterial stenosis was at the intracranial segment. The Kaplan-Meier survival analyses for the study population are shown in Figure 4. Due to the small sample size, only a univariate analysis was performed to examine the prognostic variables for recurrent stroke and mortality. The confidence intervals for recurrence and mortality were $1.54 \%-17.26 \%$ and $2.78 \%-19.82 \%$, respectively. No difference was found for hypertension, diabetes, hyperlipidemia, coronary artery disease, cigarette smoking, and previous strokes. Also, there were no differences between mono-antiplatelet therapy and dual-antiplatelet therapy.

\section{Discussion}

In this study the clinical outcomes during a 1-year follow-up period among patients with brainstem infarct due to largeartery atherothrombosis were examined. Of the stroke patients, approximately $30 \%$ die within the first year, $20 \%$ die in the early period, and approximately one-third of the

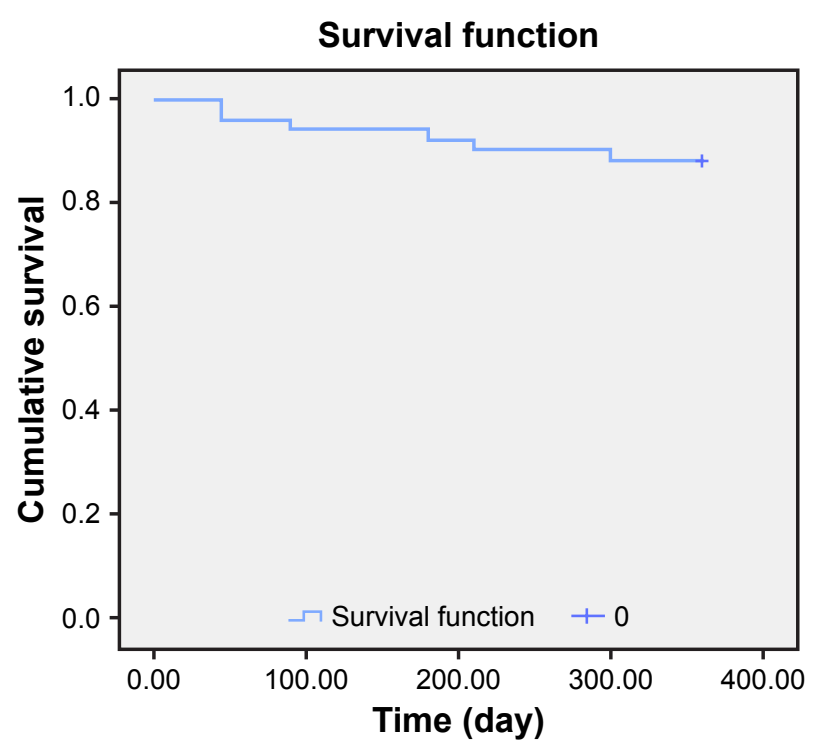

Figure 4 Kaplan-Meier survival analysis. survivors become dependent on others for their daily living activities. ${ }^{8}$ Nearly one-third to half of all ischemic strokes are associated with large-vessel atherosclerosis. This type of ischemia occurs as a result of thrombosis caused by destabilization of atheroma plaques developing in large vessels, particularly at the bifurcation points. ${ }^{9}$ Atherothrombotic lesions of the anterior circulation are generally located at the origin of the internal carotid artery and siphon, and in the posterior circulation, they are located at the origin and at the intracranial segment of the vertebral artery, as well as in the proximal basilar artery. ${ }^{10}$

Nearly $30 \%$ of all strokes are recurrent events. ${ }^{11}$ It has been reported that one-quarter of the nearly 800,000 annual stroke cases occurring in industrialized countries such as the US are recurrent strokes. ${ }^{12}$ The incidence of recurrent strokes within the subgroup of patients with ischemic strokes is similar to that observed in the general patient population with strokes. ${ }^{13}$ This condition is associated with significant mortality and economic burden, with mortality rates decreasing over decades. ${ }^{14,15}$ Even in patient populations in whom the etiology of stroke has been ascertained and secondary prevention is being implemented, recurrence rates are high. Therefore, determination of prognostic differences between subgroups of patients with stroke may influence therapeutic decisions.

Recurrence rates in patients with or without symptomatic carotid disease are well established. For instance, the reported risk of ipsilateral stroke is approximately $1 \%-3.4 \%$ among patients with $50 \%-99 \%$ asymptomatic carotid stenosis. ${ }^{16}$ The risk is particularly high in rapidly progressing plaques. The detection of silent infarcts with imaging in asymptomatic individuals is of high clinical importance with regard to the evaluation of the risk of stroke and requirement for preventive treatment. ${ }^{17}$

Generally, recurrent stroke and transient ischemic attacks have been reported to occur more frequently in posterior infarcts than in anterior infarcts, and also, the former tends to occur earlier. ${ }^{18}$ On the other hand, in a recent study by Marquardt et al involving stroke patients with $\geq 50 \%$ stenosis in vertebral and basilar arteries, the frequency of recurrent transient ischemic attacks and strokes within the first 3 months following the original event was higher as compared to those who have $\geq 50 \%$ stenosis of the carotis. ${ }^{19}$ In our study, five (10.2\%) of the 51 patients with brainstem infarcts due to large-artery thrombosis (with $\mathrm{a} \geq 50 \%$ stenosis in the vertebral or basilar artery) had recurrent events in the first 1-year period. Of these five recurrences, three occurred in the first 3 months, while the other two cases occurred after 
3 months. All recurrences involved the posterior circulation, with four resulting in death.

Several limitations of our study should be mentioned. First, our study is a single-center study with a relatively small sample size. Because it was an observational study, no standard medical treatment was administered. Due to the use of a 1.5-Tesla MRI and CT angiography, no detailed information on the plaque morphology could be provided. An MRI with higher Tesla and emboli count could yield more meaningful information.

The frequency of recurrences in patients with intracranial stenosis is controversial. In a recent study by Wang et al (the Chinese intracranial atherosclerosis study) the 1-year recurrence rates among patients with 50\%-69\%, 70\%-99\%, and total $(100 \%)$ intracranial stenosis were $3.82 \%, 5.16 \%$, and $7.27 \%$, respectively. ${ }^{20}$ In their review, Gulli et al reported that the risk of recurrence within the first 3 months in patients with vertebrobasilar artery stenosis may be elevated up to $24.6 \%$, with a significantly increased risk of recurrence particularly in patients with intracranial stenosis. ${ }^{21}$ Consistent with these data, all patients with recurrence in our study had intracranial narrowing.

The annual recurrence and mortality rate reported in patients with intracranial stenosis in the WASID study is $25 \% .^{22}$ Due to this high recurrence and mortality rate, endovascular therapy, which is a more aggressive form of treatment, was discussed and the Stenting and Aggressive Medical Management for Preventing Recurrent Stroke in Intracranial Stenosis (SAMMPRIS) study was planned. However, in that study, the annual recurrence and mortality rate in the medical treatment arm was lower than expected, ie, $12.2 \%$, and no superiority of endovascular treatment over medical treatment could be demonstrated due to the high complication rate. ${ }^{23}$ Although our study was a single-center study with a relatively small sample size, our results suggest that real-life mortality and recurrence rates are still high, particularly in patients with brainstem infarction. This suggests that aggressive treatment may be useful in patients with brainstem infarction due to large-artery atherothrombosis, who were not specifically addressed in SAMMPRIS, considering the potential for recurrence.

Although our sample size may be considered relatively smaller, the diagnosis of large-artery disease in 53 patients with an established brainstem infarct based on early-diffusion MRI was ascertained using MRA and/or CTA. Also, these patients were closely followed up for 1 year. Our results indicate that recurrence risk in patients with brainstem infarcts who have intracranial stenosis is still very high despite aggressive treatment.

\section{Disclosure}

The authors report no conflicts of interest in this work. There is no funding or financial support.

\section{References}

1. Markus HS, van der Worp HB, Rothwell PM. Posterior circulation ischaemic stroke and transient ischaemic attack: diagnosis, investigation, and secondary prevention. Lancet Neurol. 2013;12(10): 989-998.

2. Bogousslavsky J, Van Melle G, Regli F. The Lausanne stroke registry: analysis of 1,000 consecutive patients with first stroke. Stroke. 1988; 19:1083-1092.

3. Nouh A, Remke J, Ruland S. Ischemic posterior circulation stroke: a review of anatomy, clinical presentations, diagnosis, and current management. Front Neurol. 2014;5:30.

4. Compter A, van der Worp HB, Schonewille WJ, et al. VAST: vertebral artery stenting trial. Protocol for a randomised safety and feasibility trial. Trials. 2008;24(9):65.

5. Caplan L, Chung CS, Wityk R, et al. New England medical center posterior circulation stroke registry I. Methods, data base, distribution of brain lesions, stroke mechanisms, and outcomes. J Clin Neruol. 2005;1:14-30.

6. Staikov IN, Arnold M, Mattle HP, et al. Comparison of the ECST, $\mathrm{CC}$, and NASCET grading methods and ultrasound for assessing carotid stenosis. European carotid surgery trial. North American symptomatic carotid endarterectomy trial. J Neurol. 2000;247(9): 681-686.

7. Samuels OB, Joseph GJ, Lynn MJ, Smith HA, Chimowitz MI. A standardized method for measuring intracranial arterial stenosis. AJNR Am J Neuroradiol. 2000;21:643-646.

8. Kolukısa M, Asil T. Stroke. In: Işık AT, Mas R, editors. New Horizons in Geriatrik Medicine. New York: Nova science publishers; 2013:211.

9. Fisher CM, Karnes WE. Local embolism. J Neuropathol Exp Neurol. $1965 ; 24: 171-172$.

10. Fisher CM, Gore I, Okabe N, White PD. Calcification of the carotid siphon. Circulation. 1965;32:538-548.

11. Ovbiagele B. The emergency department: first line of defense in preventing secondary stroke. Acad Emerg Med. 2006;13(2):215-222.

12. American Heart Association. Heart disease and stroke statistics -2014 update: a report from the American heart association. Circulation. 2014;129:e28-e29211.

13. Lovett JK, Coull AJ, Rothwell PM. Early risk of recurrence by subtype of ischemic stroke in population-based incidence studies. Neurology. 2004;62:569-573.

14. Hong KS, Yegiaian S, Lee M, Lee J, Saver JL. Declining stroke and vascular event recurrence rates in secondary prevention trials over the past 50 years and consequences for current trial design. Circulation. 2011;123(19):2111-2119.

15. Samsa GP, Bian J, Lipscomb J, Matchar DB. Epidemiology of recurrent cerebral infarction: a Medicare claims-based comparison of first and recurrent strokes on 2-year survival and cost. Stroke. 1999;30(2):338-349.

16. Meissner I, Wiebers DO, Whisnant JP, O'Fallon WM. The natural history of asymptomatic carotid artery occlusive lesions. JAMA. 1987;258(19):2704-2707.

17. Executive Committee for the Asymptomatic Carotid Atherosclerosis Study. Endarterectomy for asymptomatic carotid stenosis. JAMA. 1995;273:1421-1428.

18. Flossmann E, Rothwell PM. Prognosis of vertebrobasilar transient ischaemic attack and minor stroke. Brain. 2003;126:1940-1954. 
19. Marquardt L, Kuker W, Chandratheva A, Geraghty O, Rothwell PM. Incidence and prognosis of $>$ or $=50 \%$ symptomatic vertebral or basilar artery stenosis: prospective population-based study. Brain. 2009;132 (pt 4):982-988.

20. Wang Y, Zhao X, Liu L, et al; CICAS Study Group. Prevalence and outcomes of symptomatic intracranial large artery stenoses and occlusions in China: the Chinese intracranial atherosclerosis (CICAS) study. Stroke. 2014;45(3):663-669.

21. Gulli G, Marquardt L, Rothwell PM, Markus HS. Stroke risk after posterior circulation stroke/transient ischemic attack and its relationship to site of vertebrobasilar stenosis: pooled data analysis from prospective studies. Stroke. 2013;44(3):598-604.
22. The Warfarin-Aspirin Symptomatic Intracranial Disease (WASID) Study Group. Prognosis of patients with symptomatic vertebral or basilar artery stenosis. Stroke. 1998;29(7):1389-1392.

23. Derdeyn CP, Chimowitz MI, Lynn MJ, et al; Stenting and Aggressive Medical Management for Preventing Recurrent Stroke in Intracranial Stenosis Trial Investigators. Aggressive medical treatment with or without stenting in high-risk patients with intracranial artery stenosis (SAMMPRIS): the final results of a randomised trial. Lancet. 2014;383(9914):333-341.

\section{Publish your work in this journal}

Neuropsychiatric Disease and Treatment is an international, peerreviewed journal of clinical therapeutics and pharmacology focusing on concise rapid reporting of clinical or pre-clinical studies on a range of neuropsychiatric and neurological disorders. This journal is indexed on PubMed Central, the 'PsycINFO' database and CAS, and is the official journal of The International Neuropsychiatric Association (INA). The manuscript management system is completely online and includes a very quick and fair peer-review system, which is all easy to use. Visit http://www.dovepress.com/testimonials.php to read real quotes from published authors.

Submit your manuscript here: http://www.dovepress.com/neuropsychiatric-disease-and-treatment-journal 